\title{
Anti-lung cancer effect of glucosamine by suppressing the phosphorylation of FOXO
}

\author{
ZHANWU YU ${ }^{1,2}$, YINGHUA $\mathrm{JU}^{3}$ and HONGXU LIU ${ }^{1}$ \\ ${ }^{1}$ Department of Thoracic Surgery, First Hospital of China Medical University, Shenyang, Liaoning 110001; ${ }^{2}$ Department of \\ Thoracic Surgery, Cancer Hospital of China Medical University Liaoning Cancer Hospital and Institute, Shenyang, \\ Liaoning 110042; ${ }^{3}$ Department of Biochemistry and Molecular Biology, College of Basic Medical Sciences, \\ China Medical University, Shenyang, Liaoning 110001, P.R. China
}

Received June 27, 2016; Accepted April 24, 2017

DOI: $10.3892 / \mathrm{mmr} .2017 .6976$

\begin{abstract}
Lung cancer is the most common cause of cancer-associated mortality worldwide, and glucosamine has the potential to exhibit antitumor activity. To reveal its anti-lung cancer mechanism, the present study investigated the effect of glucosamine on the transcriptional activity of forkhead box O (FOXO)1 and FOXO3, and associated signal transduction pathways in A549 cells. An MTT assay was performed to investigate cell viability and immunoblotting was performed to detect protein levels of FOXO1/3, phosphorylated (p)-FOXO1/3, AKT, p-AKT, extracellular signal-regulated kinase (ERK) and p-ERK, and the levels of $\beta$-O-linked N-acetylglucosamine (O-GlcNAc)-modified FOXO1 protein. Immunoprecipitation was performed to purify O-GlcNAc-modified protein prior to immunoblotting. Glucosamine inhibited FOXO1- and FOXO3-specific amino acid phosphorylation, which was correlated with its translocation from the nucleus to cytoplasm, indicating a possible anti-lung cancer mechanism of glucosamine. The present study also examined the phosphoinositide 3-kinase (PI3K)/AKT and mitogen-activated protein kinase (MAPK)/ERK pathways, which induce FOXO1- and FOXO3-specific site phosphorylation. The data showed that glucosamine suppressed the translocation of FOXO from the cytoplasm to the nucleus via glucosamine-induced O-GlcNAc modification. These observations suggested that glucosamine modulated A549 cell proliferation, possibly via O-GlcNAc modification-induced downregulation of the PI3K/AKT and MAPK/ERK pathways and their downstream signaling molecules, FOXO1 and FOXO3.
\end{abstract}

Correspondence to: Professor Hongxu Liu, Department of Thoracic Surgery, First Hospital of China Medical University, 155 Nanjingbei Street, Shenyang, Liaoning 110001, P.R. China E-mail: yuzhanwu1974@163.com

Key words: glucosamine, forkhead box O, phosphoinositide 3-kinase/AKT, mitogen-activated protein kinase/extracellular signal-regulated kinase, O-GlcNAc modification

\section{Introduction}

Forkhead transcription factors of the O class (FOXOs) are characterized by a conserved forkhead box DNA-binding domain (1). The FOXO sub-family contains four members (FOXO1, FOXO3, FOXO4 and FOXO6), which activate or repress various genes, including B-cell lymphoma 2 (Bcl-2)-interacting mediator of cell death (Bim) and Fas ligand (FasL), which are involved in apoptosis $(2,3), \mathrm{p} 27^{\mathrm{kip}}(4)$ and cyclin D (5), which are involved in cell cycle regulation, and growth arrest and DNA damage-inducible $45 \alpha$ (GADD45a), which is involved in DNA damage repair $(1-3,6)$. It has been reported that FOXO factors regulate a variety of physiological and pathological processes, and may be potential targets in tumor therapy (7). Previous studies have shown that the overexpression of FOXO3a may inhibit tumor growth in vitro and tumor size in vivo in breast cancer cells $(8,9)$. Furthermore, the cytoplasmic location of FOXO3a appears to correlate with poor survival rates in patients with breast cancer (8). As the first identified member of the FOXO subfamily, FOXO1 has been demonstrated to modulate the expression of a number of genes, including $\mathrm{p} 27^{\mathrm{kip} 1}$ and $\mathrm{p} 21^{\mathrm{cip} 1}$, which are involved in cell cycle arrest, and Bim and FasL, involved in apoptosis $(10,11)$. Growth factor stimulates activation of the phosphoinositide 3-kinase (PI3K)-AKT pathway, and induces the phosphorylation of nuclear FOXO1 at specific sites (Thr24, Ser256 and Ser319). Phosphorylated (p)-FOXO1 translocates into the cytoplasm, where it is unable to affect the expression of its target gene $(12,13)$. The AKT-mediated phosphorylation of FOXO1 on S256 facilitates interaction with the E3 ubiquitin ligase, Skp2, resulting in its ubiquitination and proteasomal degradation (14). It has been shown that the mitogen-activated protein kinase (MAPK)/extracellular signal-regulated kinase (ERK) signaling pathways can inhibit the transcriptional activity of FOXO3 by phosphorylating FOXO3 at S294, S344, $\mathrm{S} 425$. This mechanism is similar to that of the phosphorylation of FOXO1 by PI3K/AKT (9). It has been demonstrated that the PI3K/AKT and MAPK/ERK pathways are upregulated in non-small cell lung cancer (NSCLC) cell lines (15).

Glucosamine, a naturally occurring monosaccharide, acts as substrate for the biosynthesis of glycosaminoglycan and has been used to treat osteoarthritis for $>20$ years (16). 
Glucosamine has also shown potential anti-inflammatory effects by inhibiting neutrophil function, including superoxide generation, phagocytosis, granule enzyme release, chemotaxis and the expression of CD11b $(17,18)$. It has also been reported that glucosamine suppresses the expression of intercellular adhesion molecule 1 (ICAM-1) and monocyte chemoattractant protein-1 (MCP-1) in human umbilical vein endothelial cells, showing anti-atherosclerotic activity $(19,20)$. Glucosamine has a neuroprotective effect through the suppressing the production of inflammatory mediators, including interleukin- $1 \beta$, tumor necrosis factor- $\alpha$, cyclooxygenase- 2 and inducible nitric oxide synthase, inhibiting the activation of nuclear factor $(\mathrm{NF})-\mathrm{\kappa B}$ in rat brain ischemia reperfusion injury, the glucosamine-mediated induction of glucose-regulated protein 78 , which protects renal cells from hypoxia (21). Glucosamine has shown antitumor activity in vivo and in vitro $(22,23)$. The possible mechanism of its antitumor effect includes the translocation of cathepsin D and downregulation of Bcl-extra large (24), inhibition of p70S6K (25), proteasome inhibition (26), and cell cycle arrest through suppressing the expression of cyclin E (27). An increasing number of studies have shown that glucosamine can also regulate the activities of multiple proteins by suppressing specific-site amino acid phosphorylation, including p53, cyclin E and AKT $(27,28)$.

Lung cancer is the most common cause of cancerassociated mortality worldwide and NSCLC accounts for $80 \%$ of lung cancer cases (29). In the present study, the normal human bronchial epithelial (HBE) cell line and the A549 NSCLC cell line were used to examine the effect of LY294002, a PI3K-specific inhibitor, UO126, an ERK-specific inhibitor, and glucosamine on cell proliferation. The investigation focused on the effect of glucosamine on the specific-site phosphorylation of the FOXO1 protein, to attempt to elucidate the mechanism underlying in its anti-lung cancer effect.

\section{Materials and methods}

Cell culture and cytotoxicity assay. Human A549 lung adenocarcinoma cancer cells and HBE cells (Jining Shiye, Shanghai, China) were cultured in high-glucose DMEM with $10 \%$ fetal bovine serum (Gibco; Thermo Fisher Scientific, Inc., Waltham, MA, USA), penicillin (100 U/ml) and streptomycin $(100 \mu \mathrm{g} / \mathrm{ml})$. In separate experiments, LY294002 (PI3K inhibitor; $25 \mathrm{mM}$ ), UO126 (ERK inhibitor; $25 \mathrm{mM}$ ), and glucosamine sulfate (5 mM) (all from Sigma-Aldrich; Merck KGaA, Darmstadt, Germany), were added. All cells were incubated at $37^{\circ} \mathrm{C}$ in $5 \% \mathrm{CO}_{2}$.

For the cell viability assay, the HBE cells and A549 cells were seeded in 96 -well plates at $5 \times 10^{3}$ cells/well. The cells were allowed to adhere for $24 \mathrm{~h}$, and cell growth inhibition was analyzed following incubation with LY294002 $(25 \mathrm{mM})$, UO126 $(25 \mathrm{mM})$ or glucosamine sulfate $(5 \mathrm{mM})$ for $24 \mathrm{~h}$. The cells were analyzed using a 3-(4,5-dimethylthiazole-2-yl)-2,5-biphenyl tetrazolium bromide (MTT) assay (Sigma-Aldrich; Merck KGaA).

Immunoblot and immunoprecipitation analysis. The cell extracts were prepared using RIPA lysis buffer with $0.5 \%$ protease inhibitor cocktail and $1 \%$ phosphatase inhibitors. Protein samples were quantified using a Bradford Protein assay kit (Beyotime Institute of Biotechnology, Haimen, China), and $40 \mu \mathrm{g} /$ lane was separated on 10 or $12 \%$ SDS-PAGE gels and transferred for $2.5 \mathrm{~h}$ onto PVDF membranes. The blotted PVDF membranes were then probed with primary and secondary antibodies, following which the bands were visualized using enhanced chemiluminescence reagent (Sigma-Aldrich; Merck $\mathrm{KGaA})$. The membranes were blocked in 5\% not-fat dry milk and washed in TTBS $(19,20)$. Anti-FOXO1 (1:2,000; cat no. 97635; mouse), anti-p-FOXO1 (Ser 256; 1:2,000; cat no. 84192; rabbit), anti-FOXO3 (1:2,000; cat no. 2497; rabbit), anti-p-FOXO3 (Ser 294; 1:2,000; cat no. 5538; rabbit) and horseradish peroxidase (HRP)-conjugated horse anti-mouse IgG secondary antibody $(1: 1,500$; cat no. 7076) were purchased from Cell Signaling Technology, Inc. (Danvers, MA, USA). Anti-AKT (1:1,000; cat no. sc-5298; mouse), anti-p-AKT (Ser 473; 1:1,000; cat no. sc-135651; rabbit), anti-ERK (1:1,000; cat no. sc-135900; mouse), anti-p-ERK (1:1,000; cat no. sc-81492; mouse), anti-GAPDH (1:500; cat no. sc-367714; rabbit) and HRP-conjugated mouse anti-rabbit IgG secondary antibody (1:500; cat no. sc-2357) were purchased from Santa Cruz Biotechnology, Inc. (Dallas, TX, USA). Anti- $\beta$-O-linked $\mathrm{N}$-acetylglucosamine (O-GlcNAc) monoclonal antibody (1:2,000; cat no. MMS-248R; mouse) was purchased from Covance, Inc. (Princeton, NJ, USA), and HRP-conjugated goat anti-mouse $\operatorname{IgG} / \operatorname{IgM}$ (1:2,000; cat no. NBP1-75214) antibody was purchased from Novus Biologicals, LLC (Littleton, CO, USA). The bands were detected and quantified using the LAS-3000 image analyzer (Fujifilm Corporation, Tokyo, Japan). The membranes were probed with the primary antibodies overnight at $4^{\circ} \mathrm{C}$, and secondary antibodies were incubated with membranes for $1 \mathrm{~h}$ at room temperature.

For immunoprecipitation analysis (27), the cell lysates were mixed with Protein G Plus agarose for $30 \mathrm{~min}$ at $4^{\circ} \mathrm{C}$. Primary antibody (anti-O-GlcNAc monoclonal antibody; 1:50) and protein $\mathrm{G}$ agarose beads ( $40 \mu 150 \%$ bead slurry; Santa Cruz Biotechnology, Inc.) were then added to $500 \mu \mathrm{l}$ cell lysates and incubated for $2 \mathrm{~h}$ at $4^{\circ} \mathrm{C}$. Following five washes with cold lysis buffer, the immunoprecipitated samples were analyzed using western blot analysis on a $10 \%$ gel.

Statistical analysis. Data are expressed as the mean \pm standard error of the mean, and analyzed for significant differences using one-way analysis of variance with a multiple comparison test or Student's t-test (Prism 4; GraphPad Software, Inc., La Jolla, CA, USA). $\mathrm{P}<0.05$ was considered to indicate a statistically significance difference.

\section{Results}

Glucosamine inhibits lung cancer cell proliferation. To evaluate the effect of glucosamine on cell line proliferation, HBE cells and A549 cells were incubated with LY294002 ( $25 \mathrm{mM})$, UO126 $(25 \mathrm{mM})$ or $5 \mathrm{mM}$ glucosamine for $24 \mathrm{~h}$. As shown in Fig. 1, LY294002, UO126 and glucosamine inhibited the rates of cell proliferation by $\sim 15-30 \%$, showing significant inhibition and indicating the antitumor effect of glucosamine on A549 cells.

Expression of FOXO1, p-FOXO1, FOXO3 and p-FOXO3 in HBE and A549 cells. The expression levels of FOXO1 and 

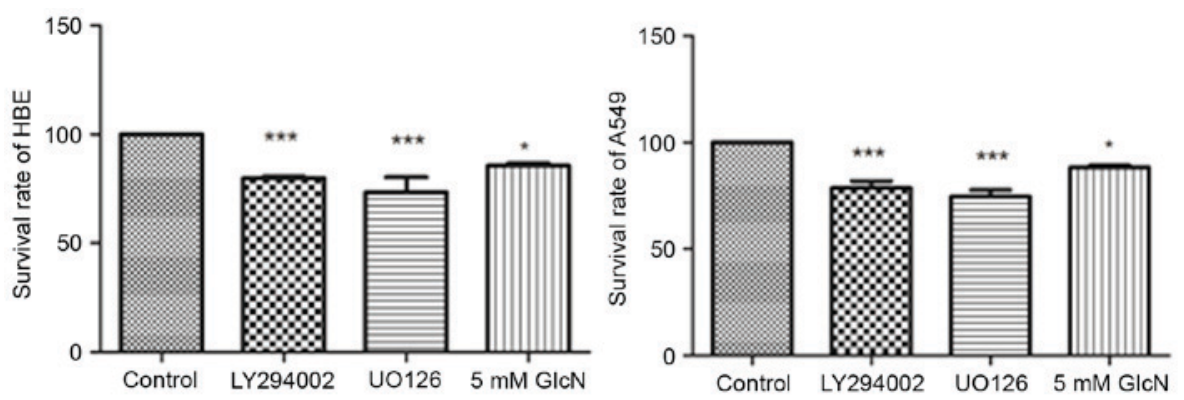

Figure 1. Effect of LY294002, UO126 and glucosamine on the proliferation of HBE and A549 cells. Cells were incubated with LY294002, UO126, or $5 \mathrm{mM}$ glucosamine for $24 \mathrm{~h}$. Viability was assessed using a 3-(4,5-dimethylthiazole-2-yl)-2,5-biphenyl tetrazolium bromide assay. Data are presented as the mean \pm standard error of the mean of six independent experiments. ${ }^{*} \mathrm{P} \leq 0.05$ and ${ }^{* * *} \mathrm{P} \leq 0.001$, compared with the untreated control. GlcN, glucosamine.
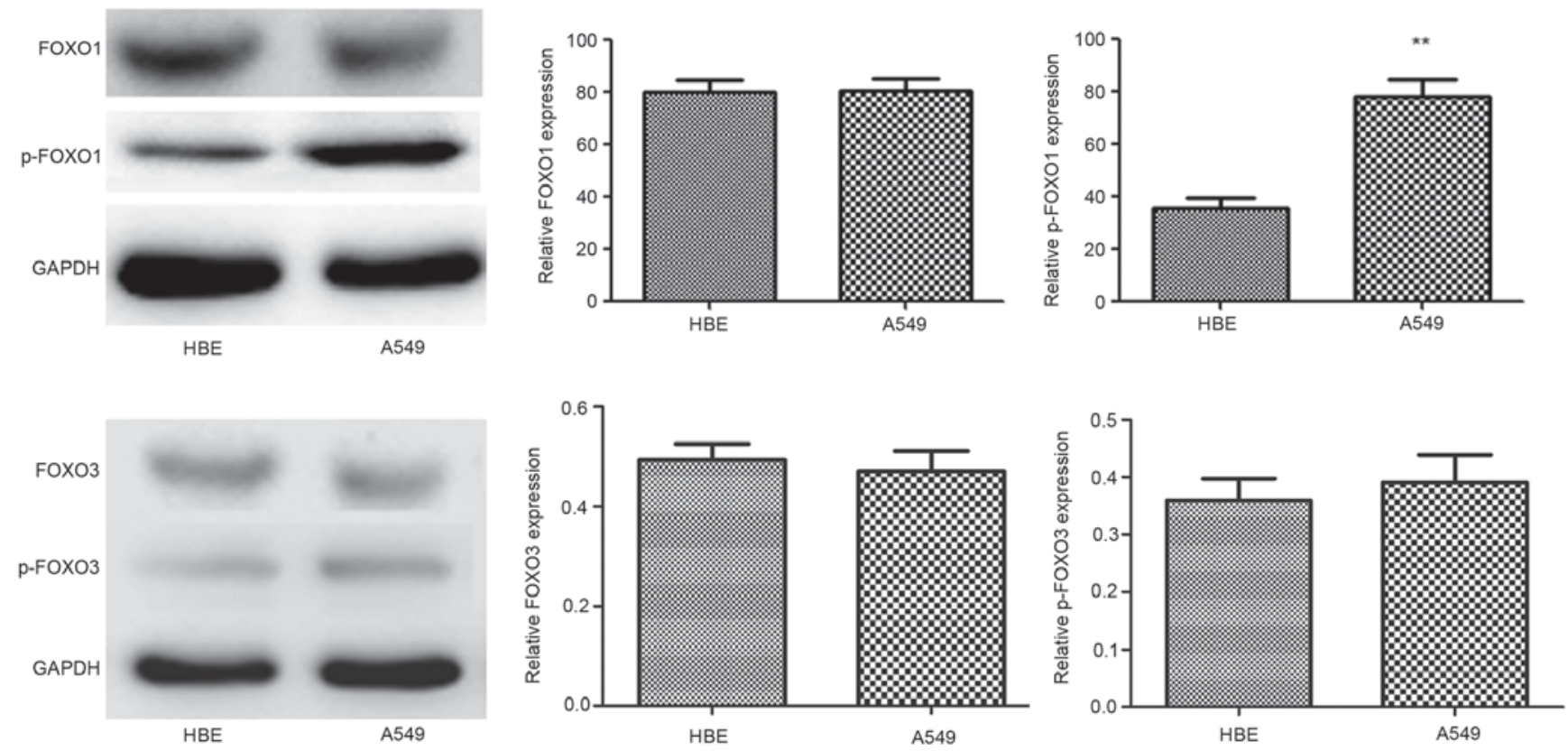

Figure 2. Expression of FOXO1, p-FOXO1, FOXO3 and p-FOXO3 in HBE and A549 cells. The expression levels of FOXO1/3, p-FOXO1/3 and GAPDH were detected using western blot analysis with the appropriate antibodies. Data are representative of at least three independent experiments. ${ }^{* *} \mathrm{P} \leq 0.01$, compared with HBE cells. FOXO, forkhead box O; p-, phosphorylated.

FOXO3 were almost identical in the two cell lines, however, p-FOXO1 was significantly upregulated in the A549 cells. Additionally, p-FOXO3 protein was also upregulated in A549 cells, however, this was not significant (Fig. 2). p-FOXO1 and $\mathrm{p}-\mathrm{FOXO} 3$ are located in the cytoplasm, and their transcriptional activities on $\mathrm{p} 27^{\mathrm{kip} 1}$ and $\mathrm{p} 21^{\text {cip1 }}$, which are involved in cell cycle arrest, and Bim and FasL, which are involved in apoptosis, are suppressed.

The protein expression levels of FOXO1, p-FOXO1, FOXO3 and p-FOXO3 were evaluated using western blot analysis in the presence of the PI3K and ERK inhibitors, LY294002 and UO126. FOXO1 and FOXO3 have previously been demonstrated to regulate the expression of a number of genes. The transcriptional activity may be regulated by multiple posttranslational modifications, for example phosphorylation, acetylation and ubiquitination (7).LY294002 and UO126 significantly inhibited the phosphorylation of FOXO1 and FOXO3, but did not affect their expression. Glucosamine significantly suppressed phosphorylation of FOXO1, and reduced the expression of FOXO3 and p-FOXO3, although this was not statistically significant for $\mathrm{FOXO} 3$ and p-FOXO3, indicating its multiple functions on translation and signal transduction (Fig. 3).

Glucosamine suppresses the phosphorylation of ERK and $A K T$. The present study evaluated the effect of glucosamine on the phosphorylation of ERK and AKT, which are two signal transduction pathways associated with cell proliferation and usually upregulated in cancer cells. As shown in Fig. 4, glucosamine inhibited the phosphorylation of ERK and AKT, indicating the potential inhibitory activity of glucosamine on these two signal transduction pathways.

Glucosamine induces O-GlcNAc modification of FOXO1. Glucosamine induced O-GlcNAc modification in the A549 cells; O-GlcNAc modification may have affected the protein phosphorylation of FOXO to alter its biological functions. The immunoprecipitation of FOXO1 in the present study confirmed that FOXO1 O-GlcNAc was modified by glucosamine (Fig. 5). 

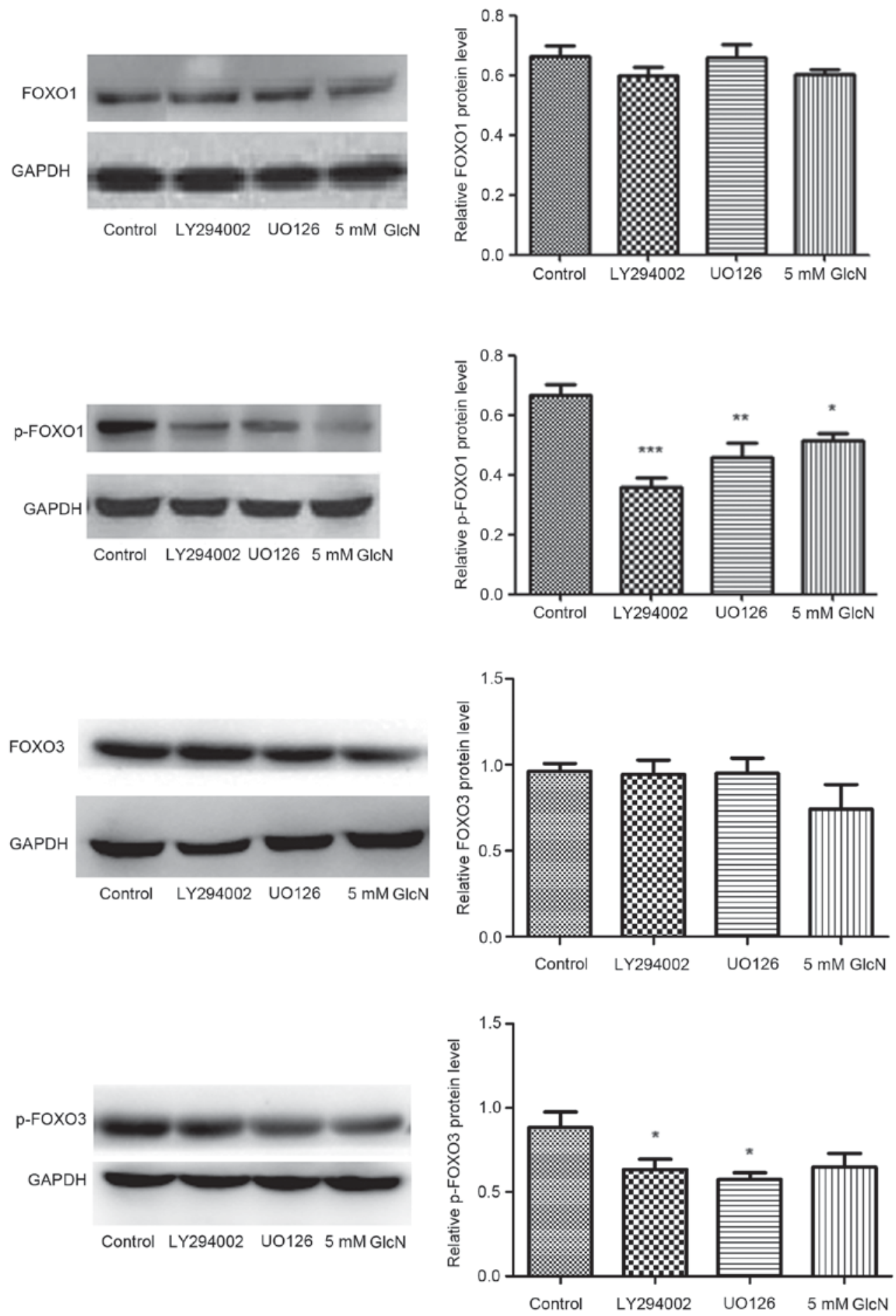

Figure 3. Effect of glucosamine on the expression of FOXO1, p-FOXO1, FOXO3, p-FOXO3. Cells were incubated with 5 mM glucosamine for 24 h. The expression levels of FOXO1/3, p-FOXO1/3 and GAPDH were detected using western blot analysis with the appropriate antibodies. Data are representative of at least three independent experiments. ${ }^{*} \mathrm{P} \leq 0.05,{ }^{* * *} \mathrm{P} \leq 0.01$ and ${ }^{* * * *} \mathrm{P} \leq 0.001$, compared with the untreated control. FOXO, forkhead box $\mathrm{O}$; $\mathrm{p}$-, phosphorylated; GlcN, glucosamine.

\section{Discussion}

Lung cancer is the leading cause of cancer-associated mortality worldwide. Although early stage disease can be cured with surgery, rates of recurrence remain high. The PI3K/AKT and MAPK/ERK signaling pathways are upregulated in lung cancer, including tissues and cell lines. The transcriptional activity of FOXO1 and FOXO3 can be regulated by multiple posttranslational modifications, including phosphorylation, acetylation and ubiquitination (12). Phosphorylated FOXO translocates into the cytoplasm, where it is unable to affect the expression of its target gene (13). Regulatory genes include $\mathrm{p} 27^{\mathrm{kip} 1}$ and $\mathrm{p} 21^{\mathrm{cip} 1}$, which are involved in cell cycle arrest, GADD45, which is involved in DNA damage repair, and Bim and FasL, which are involved in apoptosis (11). The results of the present study demonstrated that the levels of p-FOXO1 and 

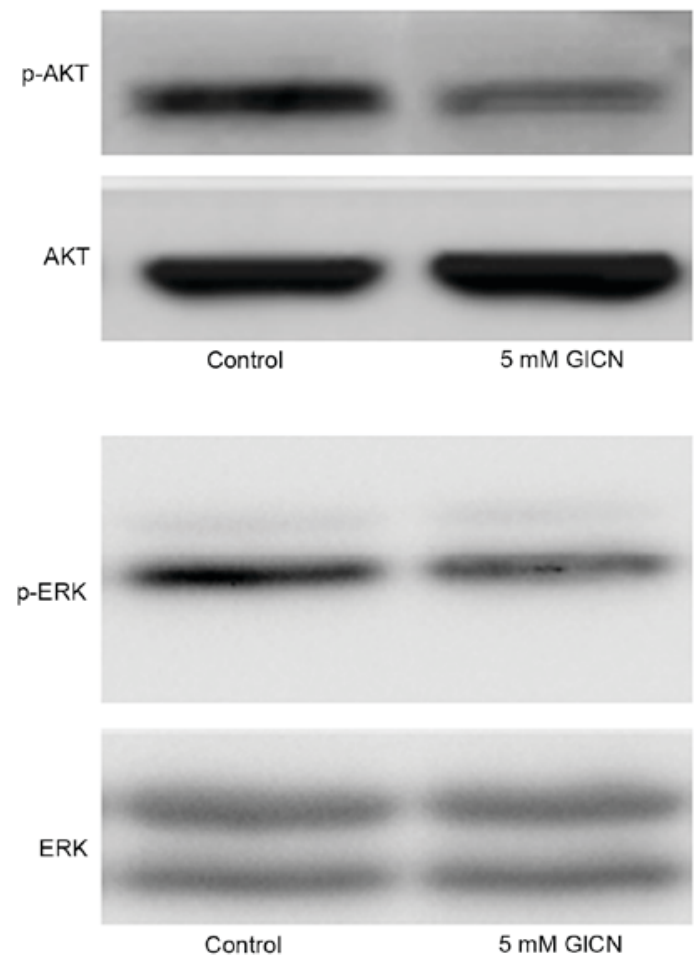

Figure 4. Effect of glucosamine on the phosphorylation of AKT and ERK. Cells were incubated with $5 \mathrm{mM}$ glucosamine for $24 \mathrm{~h}$. The expression levels of AKT and ERK, and their levels of phosphorylation were detected using western blot analysis with the appropriate antibodies. GLCN, glucosamine; ERK, extracellular signal-regulated kinase; p-, phosphorylated.

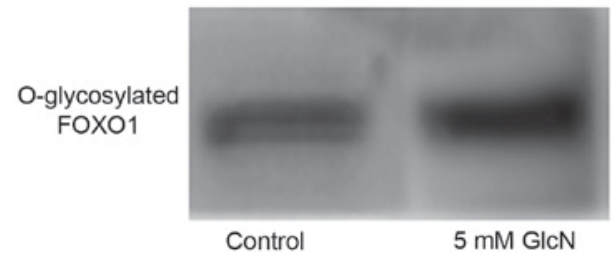

Figure 5. Glucosamine induces FOXO1 O-GlcNAc modification. Cells were incubated with or without $5 \mathrm{mM}$ glucosamine for $24 \mathrm{~h}$. FOXO1 protein was immunoprecipitated and the O-GlcNAc modification level was evaluated using western blot analysis. FOXO, forkhead box O; GlcN, glucosamine.

p-FOXO3 were upregulated in A549 cells, and that PI3K, an MAPK inhibitor, suppressed their expression. This indicated that the two signaling pathways were associated with tumorigenesis via FOXO1 and FOXO3.

Glucosamine is a naturally occurring monosaccharide, which exerts biological effects, including anti-inflammatory and antitumor effects $(2,3)$. In the present study, an MTT assay indicated the anti-lung cancer effect of glucosamine on A549 cells. The levels of protein O-GlcNAc modification of lung cancer cells induced by glucosamine were also examined, and the resulting data indicated that FOXO1 and FOXO3 O-GlcNAc modification was induced by glucosamine incubation. The glucosamine-induced protein O-GlcNAc modification sites are the same or close to sites of phosphorylation, therefore, certain proteins can be modulated by these two types of modification. In this context, it has been found that glucosamine inhibits the expression of ICAM-1 and MCP-1 by interfering with p38-MAPK- and NF- $\kappa$ B-specific site phosphorylation $(19,20)$.
The translocation of FOXO is controlled by the phosphorylation of specific amino acids. The present study investigated the suppressive effect of glucosamine on the expression of FOXO and p-FOXO, and the inhibition of phosphorylation appeared to be one of the mechanisms underlying the anti-lung cancer effect of glucosamine. The decreased protein level of FOXO3 induced by glucosamine, although not statistically significant, appeared to conflict with the anti-lung cancer effects, therefore, nucleoprotein, functioning as a transcription factor, was extracted and the protein level of FOXO was examined. The data indicated that there was no reduction in the number of nuclei, despite suppression of the protein (data not shown). The data also showed that glucosamine inhibited the PI3K/AKT and MAPK/ERK signaling pathways, which were activated in A549 cells. Our previous study indicated that the interference of the G1/S checkpoint by cyclin E was a possible mechanism underlying the anti-lung cancer effects (27). The present study showed that glucosamine inhibited lung cancer cell proliferation via multiple signal transduction pathways and by affecting the expression of various genes, including transcription, translation and post-translational degradation. In conclusion, glucosamine was found to suppress lung cancer cell proliferation, possibly by affecting the transcriptional activity of FOXO1 and p-FOXO3. The results of the present study supplement current knowledge of the mechanism underlying the anti-lung cancer effect of glucosamine and provide theoretical evidence for the clinical application of glucosamine.

\section{Acknowledgements}

This study was supported by a grant from the Department of Science and Technology of Liaoning Province (grant no. 2015020258).

\section{References}

1. Greer EL and Brunet A: FOXO transcription factors at the interface between longevity and tumor suppression. Oncogene 24: 7410-7425, 2005.

2. Finnberg N and El-Deiry WS: Activating FOXO3a, NF-kappaB and p53 by targeting IKKs: An effective multi-faceted targeting of the tumor-cell phenotype? Cancer Biol Ther 3: 614-616, 2004.

3. Tran H, Brunet A, Griffith EC and Greenberg ME: The many forks in FOXO's road. Sci STKE 2003: RE5, 2003.

4. Dijkers PF, Medema RH, Pals C, Banerji L, Thomas NS, Lam EW, Burgering BM, Raaijmakers JA, Lammers JW, Koenderman L and Coffer PJ: Forkhead transcription factor FKHR-L1 modulates cytokine-dependent transcriptional regulation of p27(KIP1). Mol Cell Biol 20: 9138-9148, 2000.

5. Schmidt M, Fernandez de Mattos S, van der Horst A, Klompmaker R, Kops GJ, Lam EW, Burgering BM and Medema RH: Cell cycle inhibition by FoxO forkhead transcription factors involves downregulation of cyclin D. Mol Cell Biol 22: 7842-7852, 2002.

6. Yang JY, Xia W and $\mathrm{Hu} \mathrm{MC}$ : Ionizing radiation activates expression of FOXO3a, Fas ligand, and Bim, and induces cell apoptosis. Int J Oncol 29: 643-648, 2006.

7. Maiese K, Chong ZZ, Shang YC and Hou J: Clever cancer strategies with FoxO transcription factors. Cell Cycle 7: 3829-3839, 2008.

8. Hu MC, Lee DF, Xia W, Golfman LS, Ou-Yang F, Yang JY, Zou Y, Bao S, Hanada N, Saso H, et al: IkappaB kinase promotes tumorigenesis through inhibition of forkhead FOXO3a. Cell 117: 225-237, 2004.

9. Yang JY, Zong CS, Xia W, Yamaguchi H, Ding Q, Xie X, Lang JY, Lai CC, Chang CJ, Huang WC, et al: ERK promotes tumorigenesis by inhibiting FOXO3a via MDM2-mediated degradation. Nat Cell Biol 10: 138-148, 2008. 
10. de Keizer PL, Packer LM, Szypowska AA, Riedl-Polderman PE, van den Broek NJ, de Bruin A, Dansen TB, Marais R, Brenkman AB and Burgering BM: Activation of forkhead box $\mathrm{O}$ transcription factors by oncogenic BRAF promotes p2 $1^{\text {cipl}}$-dependent senescence. Cancer Res 70: 8526-8536, 2010.

11. Huang $\mathrm{H}$ and Tindall DJ: Dynamic FoxO transcription factors. J Cell Sci 120: 2479-2487, 2007.

12. Yang JY and Hung MC: A new fork for clinical application: Targeting forkhead transcription factors in cancer. Clin Cancer Res 15: 752-757, 2009.

13. Vogt PK, Jiang $\mathrm{H}$ and Aoki M: Triple layer control: Phosphorylation, acetylation and ubiquitination of FOXO proteins. Cell Cycle 4: 908-913, 2005.

14. Rena G, Prescott AR, Guo S, Cohen P and Unterman TG: Rols of the forkhead in rhabdomyosarcoma (FKHR) phosphorylation sites in regulating 14-3-3 binding, transactivation and nuclear targeting. Biochem J 354: 605-612, 2001.

15. RiquelmeE,Behrens C,LinHY,Simon G,Papadimitrakopoulou V, Izzo J, Moran C, Kalhor N, Lee JJ, Minna JD and Wistuba II: Modulation of EZH2 expression by MEK-ERK or PI3K-AKT signaling in lung cancer is dictated by different KRAS oncogene mutations. Cancer Res 76: 675-685, 2016.

16. Crolle G and D'Este E: Glucosamine sulphate for the management of arthrosis: A controlled clinical investigation. Curr Med Res Opin 7: 104-109, 1980

17. Meininger CJ, Kelly KA, Li H, Haynes TE and Wu G: Glucosamine inhibits inducible nitric oxide synthesis. Biochem Biophys Res Commun 279: 234-239, 2000.

18. Hua J, Sakamoto K and Nagaoka I: Inhibitory actions of glucosamine, a therapeutic agent for osteoarthritis, on the functions of neutrophils. J Leukoc Biol 71: 632-640, 2002.

19. Ju Y, Hua J, Sakamoto K, Ogawa H and Nagaoka I: Glucosamine, a naturally occurring amino monosaccharide modulates LL-37-induced endothelial cell activation. Int J Mol Med 22: 657-662, 2008

20. Ju Y, Hua J, Sakamoto K, Ogawa H and Nagaoka I: Modulation of TNF- $\alpha$-induced endothelial cell activation by glucosamine, a naturally occurring amino monosaccharide. Int J Mol Med 22: 809-815, 2008

21. Dalirfardouei R, Karimi G and Jamialahmadi K: Molecular mechanisms and biomedical applications of glucosamine as a potential multifunctional therapeutic agent. Life Sci 152: 21-29, 2016.
22. Brasky TM, Lampe JW, Slatore CG and White E: Use of glucosamine and chondroitin and lung cancer risk in the VITamins And Lifestyle (VITAL) cohort. Cancer Causes Control 22: 1333-1342, 2011.

23. Hwang MS and Baek WK: Glucosamine induces autophagic cell death through the stimulation of ER stress in human glioma cancer cells. Biochem Biophys Res Commun 399: 111-116, 2010.

24. Wang Z, Liang R, Huang GS, Piao Y, Zhang YQ, Wang AQ Dong BX, Feng JL, Yang GR and Guo Y: Glucosamine sulfate-induced apoptosis in chronic myelogenous leukemia $\mathrm{K} 562$ cells is associated with translocation of cathepsin D and downregulation of Bcl-xL. Apoptosis 11: 1851-1860, 2006.

25. Oh HJ, Lee JS, Song DK, Shin DH, Jang BC, Suh SI, Park JW, Suh MH and Baek WK: D-glucosamine inhibits proliferation of human cancer cells through inhibition of p70S6K. Biochem Biophis Res Commun 360: 840-845, 2007.

26. Liu BQ, Meng X, Li C, Gao YY, Li N, Niu XF, Guan Y and Wang HQ: Glucosamine induces cell death via proteasome inhibition in human ALVA41 prostate cancer cell. Exp Mol Med 43: 487-493, 2011.

27. Ju Y, Yu A, Sun X, Wu D and Zhang H: Glucosamine, a naturally occurring amino monosaccharide, inhibits A549 and H446 cell proliferation by blocking G1/S transition. Mol Med Rep 8: 794-798, 2013.

28. Ozcan S, Andrali SS and Cantrell JE: Modulation of transcription factor function by O-GlcNAc modification. Biochim Biophys Acta 1799: 353-364, 2010.

29. Zheng Y, Jaklitsch MT and Bueno R: Neoadjuvant therapy in non-small cell lung cancer. Surg Oncol Clin N Am 25: 567-584, 2016. 